\title{
5-(4-Hydroxy-2,3,5-trimethylbenzylidene) thiazolidine-2,4-dione attenuates atherosclerosis possibly by reducing monocyte recruitment to the lesion
}

\author{
Jae-Hoon Choi ${ }^{1,2 *}$, Jong-Gil Park ${ }^{2,3 *}$, \\ Hyung Jun Jeon ${ }^{2}$, Mi-Sun Kim ${ }^{4}$, Mi-Ran Lee ${ }^{2}$, \\ Mi-Ni Lee ${ }^{2}$, SeongKeun Sonn ${ }^{2}$, Jae-Hong Kim ${ }^{5}$, \\ Mun Han Lee ${ }^{3}$, Myung-Sook Choi ${ }^{6}$, \\ Yong Bok Park ${ }^{7}$, Oh-Seung Kwon, \\ Tae-Sook Jeong ${ }^{9}$, Woo Song Lee ${ }^{10}$, Hyun Bo Shim², \\ Dong Hae Shin ${ }^{4}$ and Goo Taeg $\mathrm{Oh}^{2,11}$
}

${ }^{1}$ Department of Life Science

College of Natural Sciences

Hanyang University

Seoul 133-791, Korea

${ }^{2}$ Division of Life and Pharmaceutical Sciences

Ewha Womans University

Seoul 120-750, Korea

${ }^{3}$ Department of Veterinary Biochemistry

College of Veterinary Medicine

Seoul National University

Seoul 151-742, Korea

${ }^{4}$ Division of Life and Pharmaceutical Sciences

College of Pharmacy

Ewha Womans University

Seoul 120-750, Korea

${ }^{5}$ School of Life Sciences and Biotechnology

Korea University

Seoul 136-701, Korea

${ }^{6}$ Department of Food Science and Nutrition

${ }^{7}$ Department of Genetic Engineering

Kyungbook National University

Daegu 702-701, Korea

${ }^{8}$ Bioanalysis and Biotransformation Research Center

Korea Institute of Science and Technology

Seoul 136-791, Korea

${ }^{9}$ National Research Laboratory of Lipid Metabolism

\& Atherosclerosis

KRIBB

Daejeon 305-806, Korea

${ }^{10}$ Bioindustry Technology Research Center KRIBB

Jeongeup 580-185, Korea

${ }^{11}$ Corresponding author: Tel, 82-2-3277-4253;

Fax, 82-2-3277-3760; E-mail, gootaeg @ ewha.ac.kr

${ }^{*}$ These authors contributed equally to this work.

http://dx.doi.org/10.3858/emm.2011.43.8.053
Accepted 20 June 2011

Available Online 21 June 2011

Abbreviations: 5-LOX, 5-lipoxygenase; BHB-TZD, 5-(3,5-ditert-butyl-4-hydroxybenzylidene) thiazolidin-2,4-dione; COX, cyclooxygenase; HMB-TZD, 5-(4-hydroxy-2,3,5-trimethylbenzylidene) thiazolidin-2,4-dione; ICAM-1, intercellular adhesion molecule-1; Ldlr, low density lipoprotein receptor; TNF- $\alpha$, tumor necrosis factor-alpha; VCAM-1, vascular cell adhesion molecule-1

\begin{abstract}
A variety of benzylidenethiazole analogs have been demonstrated to inhibit 5-lipoxygenase (5-LOX). Here we report the anti-atherogenic potential of 5-(4-hydroxy-2,3,5-trimethylbenzylidene) thiazolidin-2,4-dione (HMB-TZD), a benzylidenethiazole analog, and its potential mechanism of action in LDL receptor-deficient $\left(\mathrm{LdII}_{\mathrm{r}}^{-1}\right)$ mice. HMB-TZD Treatment reduced leukotriene $\mathrm{B}_{4}\left(\mathrm{LTB}_{4}\right)$ production significantly in RAW264.7 macrophages and SVEC4-10 endothelial cells. Macrophages or endothelial cells pre-incubated with HMB-TZD for $\mathbf{2} \mathrm{h}$ and then stimulated with lipopolysaccharide or tumor necrosis factor-alpha (TNF- $\alpha$ ) displayed reduced cytokine production. Also, HMB-TZD reduced cell migration and adhesion in accordance with decreased proinflammatory molecule production in vitro and ex vivo. HMB-TZD treatment of 8-week-old male $L d l r^{-/}$mice resulted in significantly reduced atherosclerotic lesions without a change to plasma lipid profiles. Moreover, aortic expression of pro-atherogenic molecules involved in the recruitment of monocytes to the aortic wall, including TNF- $\alpha$, MCP-1, and VCAM-1, was downregulated. HMB-TZD also reduced macrophage infiltration into atherosclerotic lesions. In conclusion, HMB-TZD ameliorates atherosclerotic lesion formation possibly by reducing the expression of proinflammatory molecules and monocyte/macrophage recruitment to the lesion. These results suggest that HMB-TZD, and benzylidenethiazole analogs in general, may have therapeutic potential as treatments for atherosclerosis.
\end{abstract}

Keywords: antioxidants; arachidonate 5-lipoxygenase; atherosclerosis; endothelial cells; macrophages 


\section{Introduction}

Cyclooxygenase (COX) and 5-lipoxygenase (5-LOX) produce prostaglandins, thromboxanes, and leukotrienes (LTs) from arachidonic acid. These metabolites have been shown to play important roles in inflammation, thrombosis, allergy, and cancer (Vila, 2004). 5-LOX is a key enzyme involved in the production of proinflammatory LTs and has been linked to the pathogenesis of various inflammatory disorders (Vila, 2004). LTs are produced by cells critical to the inflammatory response, namely macrophages, neutrophils, eosinophils, mast cells, and endothelial cells (Lewis et al., 1990; Zou et al., 2009; Kim et al., 2010). In response to a diversity of immune and inflammatory stimuli, LTs are secreted into the extracellular space where they bind receptors, including $B L T_{1}$, $\mathrm{BLT}_{2}, \mathrm{CysLT}_{1}$, and $\mathrm{CysLT}_{2}$, and induce inflammation (Yokomizo et al., 1997, 2000; Vila, 2004; Lötzer et al., 2007; Sánchez-Galán et al., 2009).

Atherosclerosis is a chronic inflammatory vascular disease involving inflammatory mediators, monocytes, endothelial cells, and smooth muscle cells (Ross, 1999). In atherosclerotic lesions, the number of 5-LOX-expressing cells, which are primarily macrophages, is markedly increased (Spanbroek et al., 2003). In fact, a polymorphism in the 5-LOX promoter has been linked to atherosclerosis (Dwyer et al., 2004). Therefore, inhibition or genetic ablation of the 5-LOX pathway has been reported to decrease the size of atherosclerotic lesions by reducing the production of inflammatory arachidonic acid metabolites such as $\mathrm{LTB}_{4}$ (Mehrabian et al., 2002; Heller et al., 2005).

Due to their strong anti-inflammatory properties, modifiers of the 5-LOX pathway have been considered potential therapeutics for treating cardiovascular disease (Funk, 2005). Many multisubstituted benzylidenethiazole analogs derived from 2,6-di-tert-butylphenol have been found to reduce inflammatory processes in vivo and in vitro by inhibiting both COX and 5-LOX (Unangst et al., 1994). Previously, we reported that one such analog, 5-(3,5-di-tert-butyl-4-hydroxybenzylidene) thiazolidin-2,4-dione (BHB-TZD), also possesses strong anti-atherogenic activity (Choi et al., 2010). In this study, we examined the anti-atherogenic activity of another benzylidenethiazole analog, 5(4-hydroxy-2,3,5-trimethylbenzylidene) thiazolidin2,4-dione (HMB-TZD). Interestingly, although the structure of HMB-TZD is highly similar to that of BHB-TZD, HMB-TZD inhibits 5-LOX strongly but blocks COX weakly. Moreover, HMB-TZD exhibits anti-oxidative activity, preventing oxidative modification of low-density lipoprotein (LDL) (Jeong et al., 2004). These characteristics of HMB-TZD prompted us to investigate the effect of this analog on atherogenesis and its mechanism of action in $L d l r^{-1-}$ mice.

\section{Results}

\section{A potent binding mode of HMB-TZD in the catalytic channel of 5-LOX}

To elucidate a potential mode of binding of HMB-TZD to the active site of mouse 5-LOX, an in silico docking experiment was performed. Since a crystal structure of mouse 5-LOX is unavailable, we modeled this enzyme on the crystal structure of 8R-LOX from P. homomalla (Neau et al., 2009) using MODELLER. The root-mean-square deviation of $577 \mathrm{C} \alpha$-atom pairs between the final model of mouse 5-LOX and 8R-LOX is $0.340 \AA$, suggesting that these structures are similar. Our model of the structure of mouse 5-LOX consists of an $\mathrm{N}$-terminal C2-like domain and a larger C-terminal catalytic domain composed mostly of $\alpha$-helices. Within the center of the catalytic domain is a putative arachidonate binding channel connected to the catalytic iron as found in other lipoxygenases (Neau et al., 2009). Our docking experiment reveals that HMB-TZD binds to the catalytic channel of 5-LOX (Figures $1 \mathrm{~A}$ and 1B). The hydrophobic HMB moiety of HMB-TZD is positioned in the hydrophobic portion of the channel lined by Phe170, Phe178, Tyr182, Ile407, Ala411, Leu415, and Leu608. In addition, one oxygen atom of the HMB moiety forms a hydrogen bond with the nitrogen atom of the Ala411 backbone. The polar TZD moiety is located adjacent to the catalytic iron and is stabilized by hydrogen bonding between one of its oxygen atoms and His373 and Asn408. The catalytic iron is coordinated by the side chains of four amino acids (i.e., His368, His373, His551, and Asn555) and the main carboxylate of 1674 located in the C-terminus. Interestingly, these results demonstrate that one oxygen atom of the TZD moiety occupies the sixth position of the catalytic iron with an approximate distance of 3.17 $\AA$. In the purple oxygenase structure, the peroxide crucial for the catalytic activity of the enzyme occupies the sixth ligand position in the iron coordination sphere (Skrzypczak-Jankun et al., 2001). Structural comparison of several soybean 3-LOX complex structures with inhibitors such as 4-nitrocatechol (1NO3), 4-hydroperoxy-2-methoxyphenol (1HU9), protocatechuic acid (1N8Q), and epigallocathechin (1JNQ) showed a similar mode of binding between their analogous oxygen atom to the catalytic iron. Therefore, the mechanism under- 
A<smiles>Cc1cc(/C=C2\SC(=O)NC2=O)cc(C)c1O</smiles>

B

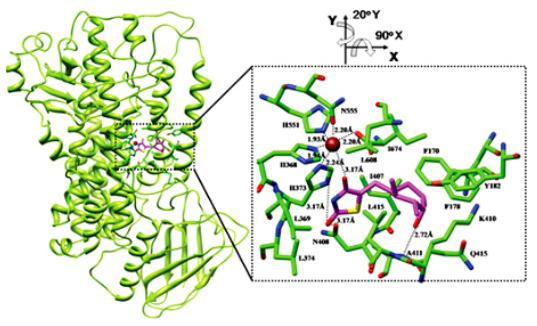

\section{C}

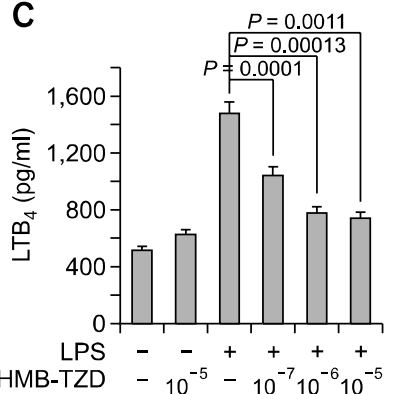

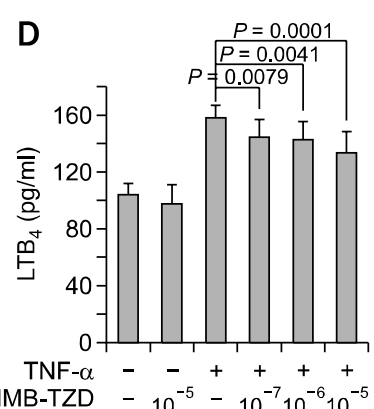

E

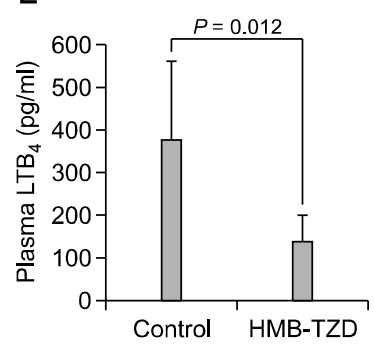

Figure 1. HMB-TZD reduces $\mathrm{LTB}_{4}$ production in vivo and in vitro. (A) Structure of HMB-TZD. (B) HMB-TZD embedded in the catalytic channel of 5-LOX. The best position for HMB-TZD within the 5-LOX catalytic channel as modeled from the docking experiment was drawn using Chimera. The zoomed image shows surrounding residues interacting with HMB-TZD. 5-LOX is colored in green and HMB-TZD in pink. The residues are represented by ball-and-stick models with yellow representing sulfur, blue for nitrogen, and red for oxygen. (C and $D)$ The concentrations of LTB $_{4}$ in RAW264.7 macrophage (C) and SVEC4-10 endothelial cell (D) culture supernatant were measured by ELISA. RAW264.7 macrophages and SVEC4-10 endothelial cells were pre-incubated with HMB-TZD $\left(10^{-5}, 10^{-6}\right.$, or $\left.10^{-7} \mathrm{M}\right)$ for $2 \mathrm{~h}$, and then stimulated with LPS $(1 \mu \mathrm{g} / \mathrm{ml})$ or TNF- $\alpha(20 \mathrm{ng} / \mathrm{ml})$ for $24 \mathrm{~h}$, respectively. (E) Effect of HMB-TZD treatment on the plasma LTB 4 level in $L d r^{-1 .}$ mice.

lying HMB-TZD inhibition of 5-LOX may be similar to these soybean 3-LOX inhibitors.

\section{HMB-TZD reduced $\mathrm{LTB}_{4}$ production in vitro and in vivo}

To determine the effect of HMB-TZD on arachidonic acid metabolite production, we first assessed the effect of the analog on the production of $\mathrm{LTB}_{4}$, a representative proatherogenic arachidonic acid metabolite produced by the 5-LOX pathway. In RAW264.7 macrophages and SVEC4-10 endothelial cells, lipopolysaccharide (LPS) or tumor necrosis factor-alpha (TNF- $\alpha$ ) stimulated $\mathrm{LTB}_{4}$ production was reduced significantly by HMB-TZD treatment in a dose-dependent manner, respectively (Figures $1 C$ and 1D). To examine the effect of HMB-TZD on plasma $\mathrm{LTB}_{4}$ levels, Ldlr knockout $\left(L d l r^{-1}\right)$ mice were fed a western diet supplemented with $1 \%$ (w/w) HMB-TZD. After 8 weeks, the $\mathrm{LTB}_{4}$ level was $63 \%$ lower in HMB-TZD-treated animals than in control mice (Figure 1E). Since many derivatives of 2,6-di-tert-butylphenol have been shown to have an inhibitory effect on COX (Unangst et al., 1994), we measured plasma prostaglandin E2 $\left(\mathrm{PGE}_{2}\right)$ levels to assess whether HMB-TZD also inhibits the COX pathway in vivo. We found no significant difference between the control and HMB-TZD- treated groups $(311 \pm 36$ vs. $325 \pm 21 \mathrm{pg} / \mathrm{ml}$, respectively). Taken together, our data demonstrate that $\mathrm{HMB}-\mathrm{TZD}$ reduces $\mathrm{LTB}_{4}$ production effectively in $L d l r^{-/}$mice.

\section{HMB-TZD reduced atherosclerotic lesion formation in $\mathrm{LdII}^{-1-}$ mice}

Initially, to investigate the pharmacokinetics of HMB-TZD, Ldlr $r^{-1}$ mice were administered $3 \mathrm{mg}$ of HMB-TZD once orally. This dose was equivalent to the daily amount ingested from the diet used in this study (i.e., 1\% w/w HMB-TZD as mentioned above). The plasma HMB-TZD level in the treated animals increased up to $1.9 \times 10^{-6} \mathrm{M}$ at $2 \mathrm{~h}$ and then decreased dramatically, indicating that HMB-TZD is metabolized and excreted normally.

To determine the effect of HMB-TZD on the development of atherosclerosis, we analyzed atherosclerotic lesions in aortic vessels from animals that were fed the western diet with and without HMB-TZD for 8 weeks. HMB-TZD supplementation did not affect plasma concentrations of total cholesterol, triglyceride, HDL- cholesterol, and LDL-cholesterol (Supplemental Data Figure S1). Necropsy and histopathological examinations did not reveal notable lesions in the parenchymal organs of both groups of mice (data not shown). In 
A
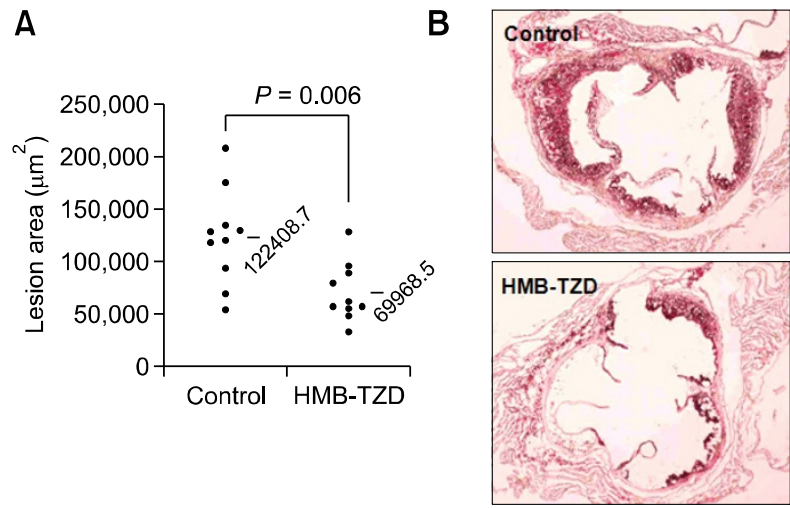

Figure 2. HMB-TZD treatment reduced fatty streak lesions in $L d l r^{-r}$ mice (A) The mean size of fatty streak lesions in HMB-TZD-treated $(n=10)$ and control $(n=10)$ mice. (B) Representative oil red $O$ staining of atherosclerotic lesions in control and HMB-TZD-treated mice.

addition, daily food intake and body weight gain was similar between the groups (data not shown). Measurement of the lesion size by computerassociated morphometry revealed that the mean lesion area of the aortic sinus in HMB-TZD-treated mice was smaller than that of control animals by $43 \%$ (Figures 2A and 2B).

Reactive oxygen species play an important role in atherogenesis through multiple mechanisms, including LDL oxidation. The lipid peroxidation product, malondialdehyde (MDA), may also be critical to atherogenesis. Previously, HMB-TZD had been reported to reduce LDL oxidation (Jeong et al., 2004). To determine the antioxidative activity of HMB-TZD, the levels of plasma lipid peroxide and lesional superoxide were measured. Although not statistically significant, the average lipid peroxide and lesional superoxide levels were lower in the HMB-TZD-treated group (Supplemental Data Figure S2).

\section{HMB-TZD reduced the expression of vascular proatherogenic genes in $\mathrm{LdIr}^{-1 /}$ mice}

To understand the mechanism underlying the antiatherogenic activity of HMB-TZD, we measured the expression of atherosclerosis-related genes, including $M C P-1, V C A M-1$, and TNF- $\alpha$, in the aortas of HMB-TZD-treated and control mice. Using real-time reverse transcription-polymerase chain reaction (RT-PCR), we found that MCP-1 and VCAM-1 mRNAs were decreased by $75 \%$ and $41 \%$, respectively, in the HMB-TZD-treated group compared to control animals (Figure $3 A$ ). In addition, TNF- $\alpha$ mRNA and plasma TNF- $\alpha$ levels were also reduced significantly by HMB-TZD administration (Figures $3 \mathrm{~A}$ and $3 \mathrm{~B}$ ).
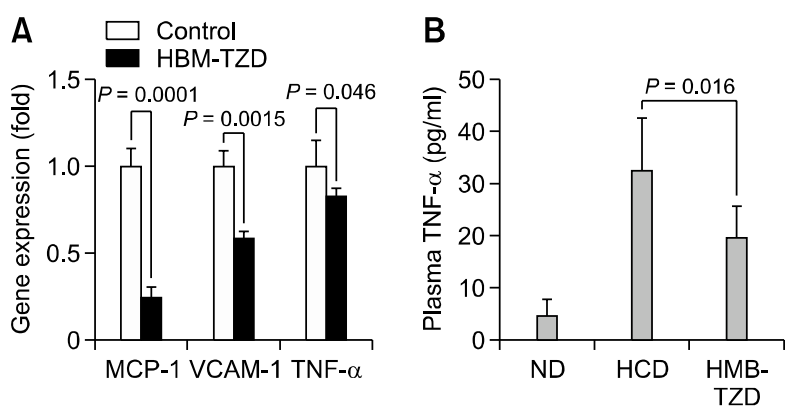

C

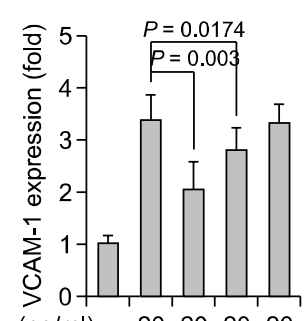

TNF- $\alpha(\mathrm{ng} / \mathrm{ml})-\begin{array}{llll}20 & 20 & 20 & 20\end{array}$ HMB-TZD (M) - $\quad 10^{-5} 10^{-6} 10^{-7}$

D

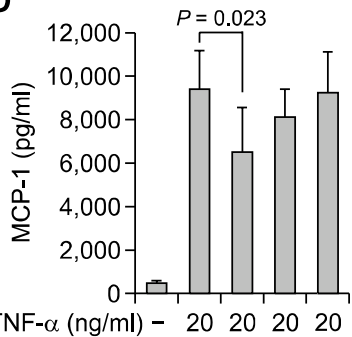

TNF- $\alpha(\mathrm{ng} / \mathrm{ml})-20202020$

Figure 3. Effect of HMB-TZD on inflammatory gene expression in the aorta and endothelial cells. Total RNA was isolated from five pooled thoracic aortas from control and HMB-TZD-treated mice. (A) The expression of MCP-1, VCAM-1, and TNF- $\alpha$ mRNA was quantitated by real-time RT-PCR and normalized to the expression of GAPDH. Each PCR reaction was performed in triplicate, and gene expression levels and standard errors were determined. (B) Reduction in plasma TNF- $\alpha$ level in HMB-TZD-treated animals. (C, D) The effect of HMB-TZD on VCAM-1 expression and MCP-1 production in HUVECs stimulated with TNF- $\alpha$. HUVECs were pre-treated with HMB-TZD at the indicated concentration for $2 \mathrm{~h}$, and then stimulated with $20 \mathrm{ng} / \mathrm{ml}$ of TNF- $\alpha$ for $24 \mathrm{~h}$.

Since HMB-TZD treatment reduces VCAM-1 expression in the aortic wall, we investigated whether this compound affects cultured endothelial cells. Our data demonstrate that HMB-TZD reduced VCAM-1 expression significantly in TNF- $\alpha$ stimulated endothelial cells in a dose-dependent manner. Moreover, MCP-1 production was decreased (Figures $3 \mathrm{C}$ and $3 \mathrm{D}$ ), indicating that $\mathrm{HMB}$ TZD reduced pro-inflammatory gene expression directly in endothelial cells.

\section{HMB-TZD inhibited monocyte adhesion to endothelium and transmigration}

Since HMB-TZD reduced the production of inflammatory molecules in cytokine-stimulated endothelial cells directly, we performed in vitro cell migration and adhesion assays, as well as ex vivo adhesion assays using aortic organ culture. These studies revealed that monocyte adhesion and migration diminished significantly with HMB-TZD treatment in vitro (Figures $4 \mathrm{~A}$ and $4 \mathrm{~B}$ ). Furthermore, monocyte attachment to the aortic lumen after 
A

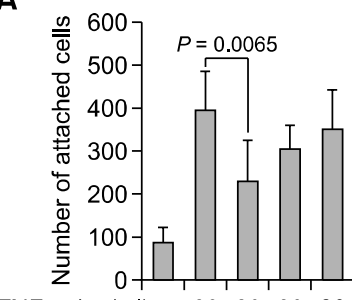

TNF- $\alpha(\mathrm{ng} / \mathrm{ml})-20202020$ HMB-TZD (M) - $-10^{-5} 10^{-6} 10^{-7}$

C
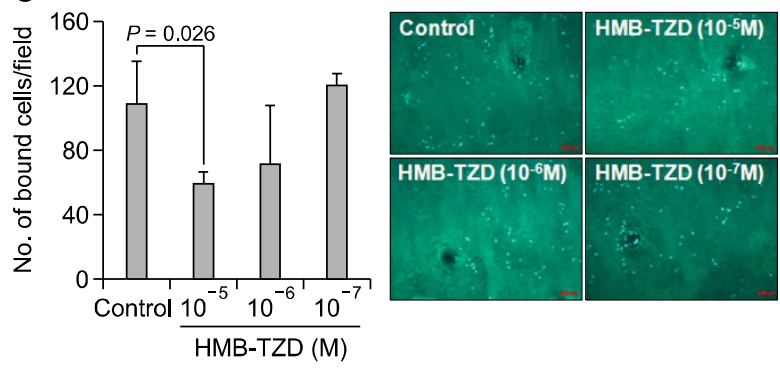

Figure 4. HMB-TZD reduced macrophage accumulation in atherosclerotic lesions. (A) Monocyte adhesion assay. HUVECs were pre-incubated with HMB-TZD $\left(10^{-5}, 10^{-6}\right.$, or $\left.10^{-7} \mathrm{M}\right)$ for $2 \mathrm{~h}$, and then stimulated with TNF- $\alpha$ for $12 \mathrm{~h}$. Next THP-1 monocytes were added to the culture. After $20 \mathrm{~min}$, unbound cells were washed and the remaining attached cells were counted in five randomly selected optical fields per well. (B) Monocyte migration assay. THP-1 monocytes were loaded into the upper chamber of Transwell plates and the lower chambers were filled with conditioned medium from HUVECs treated with HMB-TZD $\left(10^{-5}, 10^{-6}\right.$, or $10^{-7} \mathrm{M}$ ) or left untreated for $2 \mathrm{~h}$ and subsequently activated with $20 \mathrm{ng} / \mathrm{ml}$ TNF- $\alpha$ for 12 h. (C) Monocyte adhesion assay in aortic organ culture. Aortas were pre-treated for $2 \mathrm{~h}$ with $0.1 \%$ dimethyl sulfoxide or HMB-TZD $\left(10^{-5}, 10^{-6}\right.$, or $\left.10^{-7} \mathrm{M}\right)$ prior to incubation with $20 \mathrm{ng} / \mathrm{ml}$ TNF- $\alpha$ for $12 \mathrm{~h}$. After washing, aortas were incubated for 30 min with $1 \times 10^{6}$

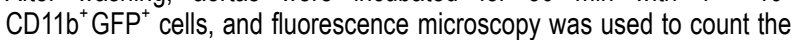
bound cells.

TNF- $\alpha$ treatment was also reduced by HMB-TZD (Figure 4C).

\section{HMB-TZD reduced macrophage accumulation in atherosclerotic lesions}

Finally, we measured the area occupied by macrophages in the aortic sinus to determine whether HMB-TZD treatment reduces monocyte recruitment to atherosclerotic lesions. HMB-TZD administration reduced the macrophage area significantly compared to untreated animals (Figure 5), suggesting that this compound affects macrophage infiltration in atherosclerotic lesions.

\section{Discussion}

In this study, we show that HMB-TZD ameliorates

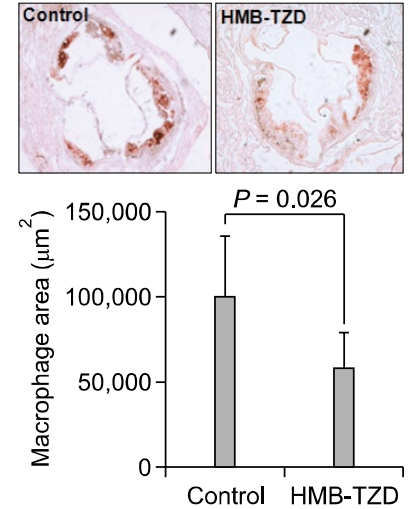

Figure 5. HMB-TZD treatment reduced macrophage infiltration into the aortic sinus of $L d l r^{-1}$ mice. Representative immunohistochemical staining of macrophages in control and HMB-TZD-treated mice are shown (upper). The graph shows the mean size of macrophage positive areas in each group (lower).

the formation of typical atheromatous lesions in $L d l r^{--}$mice without changing the plasma concentrations of total cholesterol, LDL-cholesterol, HDLcholesterol, and triglycerides. HMB-TZD also reduced the plasma level of $\mathrm{LTB}_{4}$, suggesting that this analog inhibits the 5-LOX pathway in vivo. Our data also demonstrate that this compound reduces the macrophage accumulation in atherosclerotic lesions. $\mathrm{LTB}_{4}$ is well-established as an inducer of several inflammatory processes, including chemotaxis, leukocyte adhesion to the vascular endothelium, and gene expression (Yokomizo et al., 1997, 2000; Vila, 2004; Sánchez-Galán et al., 2009). Reduced $\mathrm{LTB}_{4}$ production due to inhibition of 5-LOX activity can reduce macrophage adhesion and transmigration into the subintimal space, leading to a reduction in atherosclerotic lesion size (Aiello et al., 2002; Friedrich et al., 2003; Huang et al., 2004). Results from this study demonstrate that HMB-TZD may reduce macrophage accumulation in vessel walls, in part, by inhibiting the 5-LOX pathway, which results in downregulation of $\mathrm{LTB}_{4}$.

In this study, we show that HMB-TZD reduces $\mathrm{LTB}_{4}$ production effectively in RAW264.7 macrophages and SVEC4-10 endothelial cells. Moreover, HMB-TZD reduced plasma $\mathrm{LTB}_{4}$ levels significantly. A previous study demonstrated that benzylidenethiazole analogs derived from 2,6-di-tert-butylphenol could also inhibit COX activity (Unangst et al., 1994). Therefore, we tested the effect of HMB-TZD on $\mathrm{PGE}_{2}$ production in RAW264.7 macrophages. Our data show that HMB-TZD inhibition of $\mathrm{PGE}_{2}$ production was weak (data not shown). Furthermore, we also found that the plasma $\mathrm{PGE}_{2}$ level did not change with HMB-TZD supplementation. This result indicates that the attenuated atherosclerosis 
observed in the HMB-TZD-treated group was not mediated by COX inhibition.

In addition to anti-diabetic effects such as reducing the plasma glucose level and acting as PPAR $\gamma$ agonists, thiazolidinediones (TZDs) exhibit anti-atherogenic activity (Tontonoz and Spiegelman, 2008). HMB-TZD has a structure similar to TZDs and was demonstrated previously to react with PPAR $\gamma$ (Momose et al., 2002). Therefore, HMB-TZD is considered a PPAR $\gamma$ agonist. However, we found that HMB-TZD did not increase luciferase reporter activity when controlled by a PPAR $\gamma$-responsive element, whereas troglitazone, which was used as a positive control, increased this activity dramatically (data not shown). Furthermore, HMB-TZD supplementation did not affect plasma glucose levels (data not shown). These results indicate that HMB-TZD is not a PPAR $\gamma$ agonist, and that PPAR $\gamma$ is not involved in the attenuation of atherosclerosis by HMB-TZD.

Inhibitors of 5-LOX have been demonstrated to attenuate airway inflammation by reducing LT production (Berger et al., 2007). 5-LOX inhibitors have been used as therapeutics for asthma patients. In addition to their potent proinflammatory properties, leukotrienes have been demonstrated recently to affect atherosclerosis. Inhibitors of the 5-LOX pathway have been shown to possess anti-atherogenic activity (Aiello et al., 2002; Jawien et al., 2006). Our data support the hypothesis that inhibiting the 5-LOX pathway is a potential therapeutic strategy for treating atherosclerosis.

In conclusion, our study demonstrates that HMB-TZD reduces monocyte adhesion and infiltration. We also show that this compound attenuates atherosclerosis in LDL receptor- deficient mice. Furthermore, using in silico docking, we demonstrated that HMB-TZD can bind to the catalytic channel of 5-LOX. By reducing the $\mathrm{LTB}_{4}$ level, HMB-TZD decreased monocyte adhesion and transmigration in vitro, thereby inhibiting monocyte recruitment into the plaque. Our findings clearly support that this group of benzylidene derivatives are potential therapeutic candidates for treating atherosclerosis.

\section{Methods}

\section{Chemicals and cell culture}

HMB-TZD (Figure 1A) was obtained from the Korea Research Institute of Bioscience and Biotechnology. RAW264.7, SVEC4-10 and THP-1 cells were maintained in Dulbecco's Modified Eagles Medium (DMEM, Gibco, Grand Island, NY) supplemented with $10 \%$ fetal bovine serum. RAW264.7 macrophages were pre-incubated for 2 $h$ with HMB-TZD at concentrations that did not cause cytotoxicity as determined by the MTT assay $\left(10^{-5}, 10^{-6}\right.$, or $10^{-7} \mathrm{M}$; data not shown). Then RAW264.7 macrophages and SVEC4-10 endothelial cells were activated with 1 $\mu \mathrm{g} / \mathrm{ml}$ LPS or $20 \mathrm{ng} / \mathrm{ml}$ TNF- $\alpha$ for $24 \mathrm{~h}$. The level of LTB 4 in tissue culture supernatants from these cultures was subsequently measured by enzyme-linked immunosorbent assay (ELISA). Human umbilical vein endothelial cells (HUVECs) were purchased from Clonetics (BioWhittaker, Walkersville, MD) and maintained in EGM-2 Bullet kit media (BioWhittaker) at $37^{\circ} \mathrm{C}$ and $5 \% \mathrm{CO}_{2}$. All HUVECs used in this study were passaged 3 to 7 times.

\section{Model building and evaluation}

The primary sequence of mouse arachidonate 5-LOX consists of 674 amino acids. The Plexaura homomalla 8R-lipoxygenase (8R-LOX) sequence exhibiting 60\% homology with 5-LOX was obtained selected using BLAST (http://blast.ncbi.nlm.nih.gov) for use as a template. The 3D structure coordinates of 8R-LOX (PDB ID: 3DY5) were obtained from the Brookhaven Protein databank. MODELLER 9v8 (Eswar et al., 2006) was used to build a computational homology model of 5-LOX that satisfies spatial restraints. The input files for MODELLER were the pdb file of the template, the alignment file of the template and target sequences, and Python command script files. Energy-minimized models were produced using CHARMM energy parameters. The most reasonable model was selected based on evaluation and quality assessment of the stereochemistry of the model by PROCHECK (Laskowski et al., 1996). ProSa (Sippl, 1993) generating energy graphs based on interactions between $C \beta-C \beta$ pairs and $C \beta$-surface was also used to check strained regions in the model.

\section{In vivo docking}

To probe the preferred binding mode of HMB-TZD to the active site of 5-LOX, in silico docking studies were performed using the modeled structure of 5-LOX. To accomplish this, the docking algorithm Surflex-Dock, which is part of the SYBYL software package (version SYBYL-X 1.1, Tripos, Inc.), was used. The active site of 5-LOX was defined using the SYBYL structure preparation tool based on the complex structures of 3-LOX from soybean with 1NO3, 1HU9, 1N8Q, 13(S)-hydroperoxy-9(Z), 11(E)-octadecadienoic acid, or $1 \mathrm{JNQ}$. The HMB-TZD structure drawn in PubChem Sketcher v2.4 (http://pubchem.ncbi.nlm.nih .gov) was converted to a 3D structure and energyminimized with the Gasteiger-Huckel force field and saved in the MOL format for docking.

\section{LdIIr-l mice and HMB-TZD treatment}

Eight-week-old male $L d l r^{-/}$mice $(n=20)$ were divided randomly into two equal-sized groups, control and HMB-TZD-treated. The control group was fed a western diet (CRF-1 supplemented with $0.15 \%$ cholesterol, $20 \%$ fat, and $0.05 \%$ Na-cholate, Oriental Yeast Co. Ltd., Tokyo, Japan), while the HMB-TZD-treated mice were fed the same diet supplemented with $1 \%(w / w)$ HMB-TZD. All mice were given water and food ad libitum. After 8 weeks, the mice were sacrificed, and plasma levels of various 
molecules were determined using an automatic blood chemical analyzer (HITACHI, Japan). Other parenchymal organs were harvested for histopathological examination. This investigation conforms to the Guidelines for the Care and Use of Laboratory Animals published by the United States National Institutes of Health. All animal study protocols were approved by the Institutional Animal Care and Usage Committee of Ewha Womans University (Seoul, Korea).

\section{Measurement of $\mathrm{LTB}_{4}, \mathrm{PGE}_{2}$, MCP-1, and TNF- $\alpha$ levels}

The levels of $\mathrm{LTB}_{4}, \mathrm{PGE}_{2}, \mathrm{MCP}-1$, and TNF- $\alpha$ in plasma or cell culture supernatant were quantified by ELISA (R\&D Systems, Minneapolis, MN) according to the manufacturer's instructions. Each measurement was performed in triplicate and three independent measurements were conducted.

\section{Statistical analysis}

Statistical significance between two groups was determined with the two-tailed Student's $t$ test. Analysis of variance followed by Fisher's Protected Least Significant Difference was used for comparisons within a test group. All data in this study are expressed as the mean \pm standard deviation. Values of $P<0.05$ were considered significant.

\section{Supplemental data}

Supplementary data were included with methods for establishing the pharmacokinetics of HMB-TZD in Ldlrmice, measurement of plasma lipid peroxide, measurement of superoxide in atherosclerotic lesions, histopathology, quantitative real-time RT-PCR, macrophage adhesion assay, mouse aorta isolation, and ex vivo adhesion assay and cell migration. Supplemental Data include two figures and can be found with this article online at http://e-emm.or.kr/ article/article_files/ SP-43-8-05.pdf.

\section{Acknowledgements}

This study was supported by a grant from the Korea Health 21 R\&D Project, Ministry of Health and Welfare, Republic of Korea (A090264).

\section{References}

Aiello RJ, Bourassa PA, Lindsey S, Weng W, Freeman A, Showell HJ. Leukotriene B4 receptor antagonism reduces monocytic foam cells in mice. Arterioscler Thromb Vasc Biol 2002;22:443-9

Berger W, De Chandt MT, Cairns CB. Zileuton: clinical implications of 5-Lipoxygenase inhibition in severe airway disease. Int J Clin Pract 2007;61:663-76

Choi JH, Jeon HJ, Park JG, Sonn SK, Lee MR, Lee MN, You HJ, Kim GY, Kim JH, Lee MH, Kwon OS, Nam KH, Kim HC, Jeong TS, Lee WS, Oh GT. Anti-atherogenic effect of
BHB-TZD having inhibitory activities on cyclooxygenase and 5-lipoxygenase in hyperlipidemic mice. Atherosclerosis 2010;212:146-52

Dwyer JH, Allayee H, Dwyer KM, Fan J, Wu H, Mar R, Lusis AJ, Mehrabian M. Arachidonate 5-lipoxygenase promoter genotype, dietary arachidonic acid, and atherosclerosis. N Engl J Med 2004;350:29-37

Eswar N, Webb B, Marti-Renom MA, Madhusudhan MS, Eramian D, Shen MY, Pieper U, Sali A. Comparative protein structure modeling using Modeller. Curr Protoc Bioinformatics 2006; Chapter 5:Unit 5.6

Friedrich EB, Tager AM, Liu E, Pettersson A, Owman C, Munn L, Luster AD, Gerszten RE. Mechanisms of leukotriene B4--triggered monocyte adhesion. Arterioscler Thromb Vasc Biol 2003;23:1761-7

Funk CD. Leukotriene modifiers as potential therapeutics for cardiovascular disease. Nat Rev Drug Discov 2005; 4:664-72

Heller EA, Liu E, Tager AM, Sinha S, Roberts JD, Koehn SL, Libby P, Aikawa ER, Chen JQ, Huang P, Freeman MW, Moore KJ, Luster AD, Gerszten RE. Inhibition of atherogenesis in BLT1-deficient mice reveals a role for LTB4 and BLT1 in smooth muscle cell recruitment. Circulation 2005; 112:578-86

Huang L, Zhao A, Wong F, Ayala JM, Struthers M, Ujjainwalla F, Wright SD, Springer MS, Evans J, Cui J. Leukotriene B4 strongly increases monocyte chemoattractant protein-1 in human monocytes. Arterioscler Thromb Vasc Biol 2004;24:1783-8

Jawien J, Gajda M, Rudling M, Mateuszuk L, Olszanecki R, Guzik TJ, Cichocki T, Chlopicki S, Korbut R. Inhibition of five lipoxygenase activating protein (FLAP) by MK-886 decreases atherosclerosis in apoE/LDLR-double knockout mice. Eur J Clin Invest 2006;36:141-6

Jeong TS, Kim JR, Kim KS, Cho KH, Bae KH, Lee WS. Inhibitory effects of multi-substituted benzylidenethiazolidine-2,4-diones on LDL oxidation. Bioorg Med Chem 2004;12:4017-23

Kim JM, Lee EK, Park G, Kim MK, Yokozawa T, Yu BP, Chung HY. Morin modulates the oxidative stress-induced NF-kappaB pathway through its anti-oxidant activity. Free Radic Res 2010;44:454-61

Laskowski RA, Rullmannn JA, MacArthur MW, Kaptein R, Thornton JM. AQUA and PROCHECK-NMR: programs for checking the quality of protein structures solved by NMR. J Biomol NMR 1996;8:477-86

Lewis RA, Austen KF, Soberman RJ. Leukotrienes and other products of the 5-lipoxygenase pathway. Biochemistry and relation to pathobiology in human diseases. $\mathrm{N}$ Engl J Med 1990;323:645-55

Lötzer K, Jahn S, Kramer C, Hildner M, Nüsing R, Funk CD, Habenicht AJ. 5-Lipoxygenase/cyclooxygenase-2 crosstalk through cysteinyl leukotriene receptor 2 in endothelial cells. Prostaglandins Other Lipid Mediat 2007;84:108-15

Mehrabian $M$, Allayee $H$, Wong $J$, Shi W, Wang XP, Shaposhnik Z, Funk CD, Lusis AJ. Identification of 
5-lipoxygenase as a major gene contributing to atherosclerosis susceptibility in mice. Circ Res 2002;91:120-6

Momose $\mathrm{Y}$, Maekawa T, Yamano T, Kawada M, Odaka $\mathrm{H}$, Ikeda $\mathrm{H}$, Sohda T. Novel 5-substituted 2,4-thiazolidinedione and 2,4-oxazolidinedione derivatives as insulin sensitizers with antidiabetic activities. J Med Chem 2002;45:1518-34

Neau DB, Gilbert NC, Bartlett SG, Boeglin W, Brash AR, Newcomer ME. The 1.85 A structure of an 8R-lipoxygenase suggests a general model for lipoxygenase product specificity. Biochemistry 2009;48:7906-15

Ross R. Atherosclerosis--an inflammatory disease. N Engl J Med 1999;340:115-26

Sánchez-Galán E, Gómez-Hernández A, Vidal C, MartínVentura JL, Blanco-Colio LM, Muñoz-García B, Ortega L, Egido J, Tuñón J. Leukotriene B4 enhances the activity of nuclear factor-kappaB pathway through BLT1 and BLT2 receptors in atherosclerosis. Cardiovasc Res 2009; 81:216-25

Sippl MJ. Recognition of errors in three-dimensional structures of proteins. Proteins 1993;17:355-62

Skrzypczak-Jankun E, Bross RA, Carroll RT, Dunham WR, Funk MO Jr. Three-dimensional structure of a purple lipoxygenase. J Am Chem Soc 2001;123:10814-20

Spanbroek R, Grabner R, Lotzer K, Hildner M, Urbach A, Ruhling K, Moos MP, Kaiser B, Cohnert TU, Wahlers T, Zieske A, Plenz G, Robenek H, Salbach P, Kuhn H, Radmark $\mathrm{O}$, Samuelsson B, Habenicht AJ. Expanding expression of the 5-lipoxygenase pathway within the arterial wall during human atherogenesis. Proc Natl Acad Sci USA 2003; 100:1238-43

Tontonoz P, Spiegelman BM. Fat and beyond: the diverse biology of PPARgamma. Annu Rev Biochem 2008; 77:289-312

Unangst PC, Connor DT, Cetenko WA, Sorenson RJ, Kostlan CR, Sircar JC, Wright CD, Schrier DJ, Dyer RD. Synthesis and biological evaluation of 5-[[3,5-bis(1,1-dimethylethyl)4-hydroxyphenyl]methylene]oxazoles, -thiazoles, and -imidazoles: novel dual 5-lipoxygenase and cyclooxygenase inhibitors with antiinflammatory activity. J Med Chem 1994; $37: 322-8$

Vila L. Cyclooxygenase and 5-lipoxygenase pathways in the vessel wall: role in atherosclerosis. Med Res Rev 2004; 24:399-424

Yokomizo T, Izumi T, Chang K, Takuwa Y, Shimizu T. A G-protein-coupled receptor for leukotriene B4 that mediates chemotaxis. Nature 1997;387:620-4

Yokomizo T, Kato K, Terawaki K, Izumi T, Shimizu T. A second leukotriene $\mathrm{B}(4)$ receptor, BLT2. A new therapeutic target in inflammation and immunological disorders. J Exp Med 2000;192:421-32

Zou Y, Kim DH, Jung KJ, Heo HS, Kim CH, Baik HS, Yu BP, Yokozawa T, Chung HY. Lysophosphatidylcholine enhances oxidative stress via the 5-lipoxygenase pathway in rat aorta during aging. Rejuvenation Res 2009;12:15-24 